\title{
Giant resonant enhancement of optical binding of dielectric particles
}

\author{
Evgeny N. Bulgakov, Konstantin N. Pichugin, and Almas F. Sadreev \\ Kirensky Institute of Physics, Federal Research Center KSC SB RAS, 660036 Krasnoyarsk, Russia
}

(Dated: August 13, 2020)

\begin{abstract}
Optical coupling of two identical dielectric particles gives rise to bonding and anti-bonding resonances. The latter is featured by significant narrowing of the resonant width and strong enhancement of the $Q$ factor for the high index micron size particles in subwavelength range. We consider particles shaped as spheres and disks under coaxial illumination of dual incoherent counter propagating Bessel beams. In the case of spheres we derive analytical expressions for the optical binding (OB) force which decreases as $1 / L^{2}$ for large distance $L$ between the spheres and displays two periods of oscillations. For close distances the OB force enormously increases in the resonant regime. The case of two coaxial disks owing to variation of the distance between disks and aspect ratio of each disk is featured by extremal enhancement of the $Q$ factor compared to the case of two spheres. In that case we demonstrate unprecedent enhancement of the OB force up to several decades of nano Newtons. We show that the magnitude and sign of the OB force strongly depend on the longitudinal wave vector of the Bessel beams.
\end{abstract}

PACS numbers:

\section{INTRODUCTION}

The response of a microscopic dielectric object to a light field can profoundly affect its motion. A classical example of this influence is an optical trap, which can hold a particle in a tightly focused light beam [1]. When two or more particles are present, the multiple scattering between the objects can, under certain conditions, lead to optically bound states. This is often referred to peculiar manifestation of optical forces as optical binding (OB), and it was first observed by Burns et al. on a system of two plastic spheres in water in 1989 [2]. Optical binding belongs to an interesting type of mechanical light-matter interaction between particles at micro-scale mediated by the light scattered by illuminated particles. Depending on the particle separation, OB leads to attractive or repulsive forces between the particles and, thus, contributes to the formation of stable configurations of particles. The phenomenon of OB can be realized, for example, in dual counter propagating beam configurations [3 9]. Equilibrium positions of particles are created by a very weak balance between the optical forces from the incident fields and from the scattered fields generated by the particles. Many researchers have analyzed OB force quantitatively in theory. Chaumet et al [10] and $\mathrm{Ng}$ et al [11] calculated the OB force under illumination of two counter propagating plane waves. Čižmár et al 12] presented the first theoretical and experimental study of dielectric sub-micron particle behavior and their binding in an optical field generated by interference of two counter propagating Bessel beams. Also Thanopulos et al [9, 13] numerically evaluated the OB force as a function of distance between spheres and frequency.

It is clear that excitation of the resonant modes with high $Q$ factor in dielectric structures results in large enhancement of near electromagnetic (EM) fields and respectively in extremely large EM forces proportional to squared EM fields. First, sharp features in the force spectrum, causing mutual attraction or repulsion between successive photonic crystal layers of dielectric spheres under illumination of plane wave has been considered by Antonoyiannakis and Pendry [14]. Because of periodicity of the structure each layer is specified by extremely narrow resonances which transform into the bonding and anti-boding resonances for close approaching of the layers. It was shown that the normal force acting on each layer as well as the total force acting on both layers including the optical binding force follow these resonances. It was revealed that the lower frequency bonding resonance forces push the two layers together and the higher frequency anti-bonding resonance pull them apart. Later these disclosures we reported for coupled photonic crystal slabs [15] and two planar dielectric photonic metamaterials [16] due to existence of resonant states with infinite $Q$ factor (bound states in the continuum).

However in practice we have arrays of finite number of dielectric particles which nevertheless show the $Q$ factor exceeding the $Q$ factor of individual particle by many orders in magnitude [17 - 19]. What is remarkable even two particles can demonstrate extremely high $Q$ resonant modes owing to avoided crossings. The vivid example is avoided crossing of whispering-gallery modes (WGM) in coupled microresonators which results in extremely high $Q$ factor [20, 21]. As a result an enhancement of the OB force around of hundreds of nano Newtons between coupled WGM spherical resonators takes place in applied power $1 \mathrm{~mW}[20$. However, the WGM modes with extremely high orbital momenta can be excited only in spheres with large radii of order $30 \mu \mathrm{m}$. Recently we offered a solution to the problem of large $Q$ factor in the subwavelength regime by use of two coaxial silicon disks of micron sizes. Owing to two-parametric (over the aspect ratio and distance between disks) avoided crossing of low order resonances the anti-bonding resonant mode acquires a morphology of the higher order Mie resonant mode of effective sphere with 

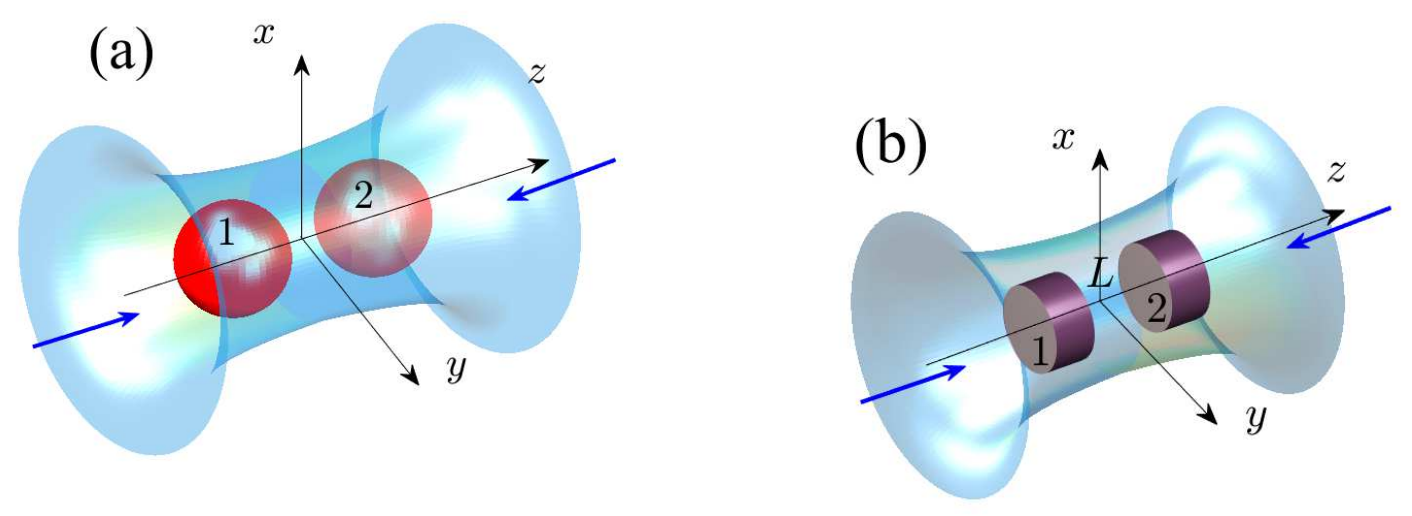

FIG. 1: Two silicon spheres (a) and disks (b) with the radius $a$, permittivity $\epsilon=15$ under illumination of two counterpropagating mutually incoherent Bessel beams with zero azimuthal index $m=0$. Light intensity of each beam $P_{0}=1 m W / \mu m^{2}$.

extremely small resonant width [22].

In addition to disks we consider silicon spheres which are subject to only one-parameter avoided crossing (the distance between spheres). As a result the spheres do not show extremely high $Q$ factors and respectively giant OB forces but have an advantage of analytical consideration of the OB forces under illumination of dual counter propagating Bessel beams. We show that two spheres demonstrate the same features of the OB force which are inherent two disks. The optical forces for single sphere were explicitly derived by Barton et al [23] in general case that allows to consider the OB force analytically for the present case of two spheres. The consideration is significantly simplified when the spherical particles are subject to beams like Gaussian or Bessel if they preserve axial symmetry. Then the binding force depends on the distance between the spheres only [5, 11, 24 27]. That allows us to derive analytical expressions for the OB force which decreases as $1 / L^{2}$ for large distances $L$ between spheres and displays two periods of oscillations as was first revealed by Karasek el al 25] numerically. When the spheres are close to each other the $\mathrm{OB}$ force enormously increases if the frequency of Bessel beams follows to the bonding or anti-bonding resonances. We show also that a magnitude and what is more interesting the sign of the OB force strongly depend on the wave number of the Bessel beams that opens additional options to arrange high index particles optically.

\section{OPTICAL BINDING FORCE OF TWO SPHERES}

In order to stabilize the spheres across to beam we use the results by Milne et al [28] that the Bessel beams strongly trap spherical particles at the symmetry axis, i.e., at $r=0$ (stable zero-force points). That justifies the calculation of the $\mathrm{OB}$ as dependent on the distance between the spheres positioned at the symmetry axis. We consider the Bessel beams with TE polarization in the simplest form with zero azimuthal index $m=0$ [25]

$$
\mathbf{E}_{i n c}(r, \phi, z)=E_{0} \mathbf{e}_{\phi} \exp \left(i k_{z} z\right) J_{1}\left(k_{r} r\right)
$$

where $J_{1}$ is an Bessel function, $k_{z}$ and $k_{r}$ are the longitudinal and transverse wave numbers, with the frequency $\omega / c=k=\sqrt{k_{r}^{2}+k_{z}^{2}}$ and $r, \phi$, and $z$ are the cylindrical coordinates, $\mathbf{e}_{\phi}$ is the unit vector of the polarization. In order to consider the $\mathrm{OB}$ force we use the approach in which two counter-propagating mutually incoherent Bessel beams were applied [3, 5] which are schematically shown in Fig. 10 (a).

The electromagnetic (EM) force is defined by the stress-tensor $T_{\alpha \beta}$ integrated over the surface elements $d S_{\beta}$ outside the particle [29, 30]

$$
\begin{gathered}
F_{\alpha}=\int T_{\alpha \beta} d S_{\beta}, \\
T_{\alpha \beta}=\frac{1}{4 \pi} E_{\alpha} E_{\beta}^{*}-\frac{1}{8 \pi} \delta_{\alpha \beta}|\mathbf{E}|^{2}+\frac{1}{4 \pi} H_{\alpha} H_{\beta}^{*}-\frac{1}{8 \pi} \delta_{\alpha \beta}|\mathbf{H}|^{2} .
\end{gathered}
$$

This problem allows analytical treatment owing to a series of the Bessel beam and scattered fields both over the vectorial spherical harmonics. Such an approach was used to find the optical forces for the case of the isolated 

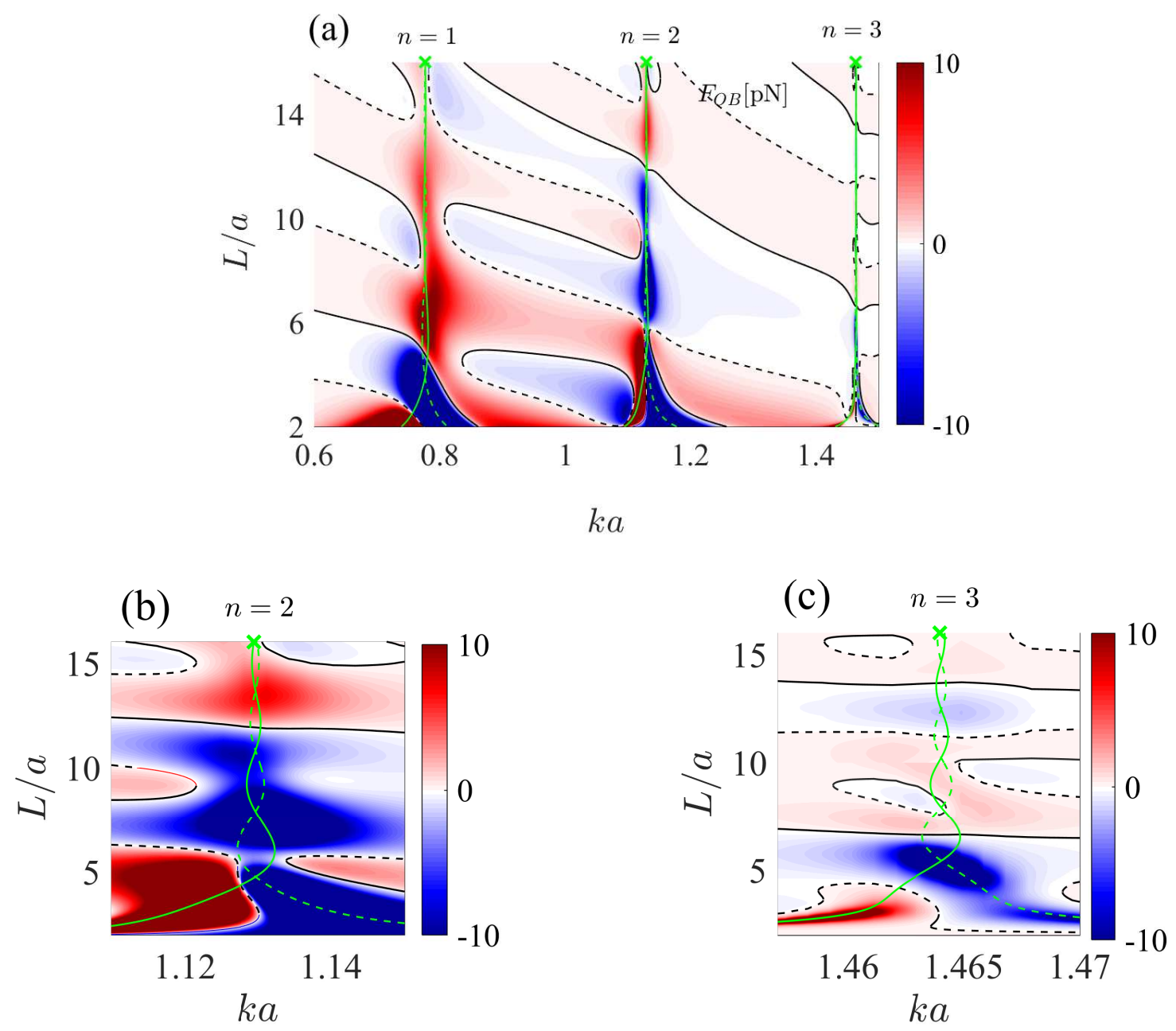

FIG. 2: The binding force between two spheres vs the frequency and distance for the dual Bessel beam of power $1 m W / \mu m{ }^{2}$ with TE polarization, $k_{z} a=1 / 2$ where $a=0.5 \mu \mathrm{m}$ is the sphere radius with $\epsilon=15$. (b) and (c) zoomed versions. The red corresponds to attractive forces and blue corresponds to repulsive OB force. Black solid (dash) lines show stable (unstable) configuration of spheres. Light green solid (dash) lines show symmetric (anti symmetric) resonant frequencies of two spheres vs the distance between. Crosses mark the Mie TE resonances in isolated dielectric sphere.

sphere 31 35]. In the case of two spheres multiple scattering theory was used to define the OB forces and calculate them numerically [5, 11, 24 27. By using this theory we performed numerical simulations of the complex resonant frequencies and binding force of two coupled spheres with focus on the dependence of the OB force on the intrinsic parameters such as the distance between spheres and external parameters such the frequency and wave number of the dual Bessel beams.

The results of calculations are presented in Figs. 2 and 3 for two values $k_{z} a=1 / 2$ and $k_{z} a=1$ of the Bessel beam (11). We show the binding force $F_{O B}=\left(F_{1 z}-F_{2 z}\right) / 2$ where the indices 1 and 2 denote the spheres where the Bessel beam incident at the left. Owing to an incoherence of the Bessel beam illuminated from the right we have the same expression for $F_{O B}=-F_{O B}$. As a result we obtain doubled value for the OB force $F_{O B}=F_{1 z}-F_{2 z}$. Strong resonant forces above $10 p N$ by absolute value in Figs. 2 and 3 are saturated by intense red (attractive) or blue (repulsive). As it was expected the OB force shows vivid resonant behavior near the Mie resonances of the individual sphere labelled by orbital index $n$ because of resonant enhancement of scattered fields. However, for variation of the distance between spheres we see a number of peculiarities. The first one is periodic oscillations when the repulsive OB force is alternating by the attractive one. Respectively, the equilibrium distances shown in Figs. 2 and 3 by solid lines follow a law $\left(k+k_{z}\right) L=2 \pi l+\phi_{0}, l=1,2,3, \ldots$ and undergo abrupt changes near the Mie resonances $k_{n}$. This period will be derived below asymptotically for large $L$ and first was predicted by Karasek el al 25]. Also in Figs. 2 and 3 we show the resonant frequencies of two spheres (even/odd relative to $z \rightarrow-z$ or bonding/antibonding) versus the distance 


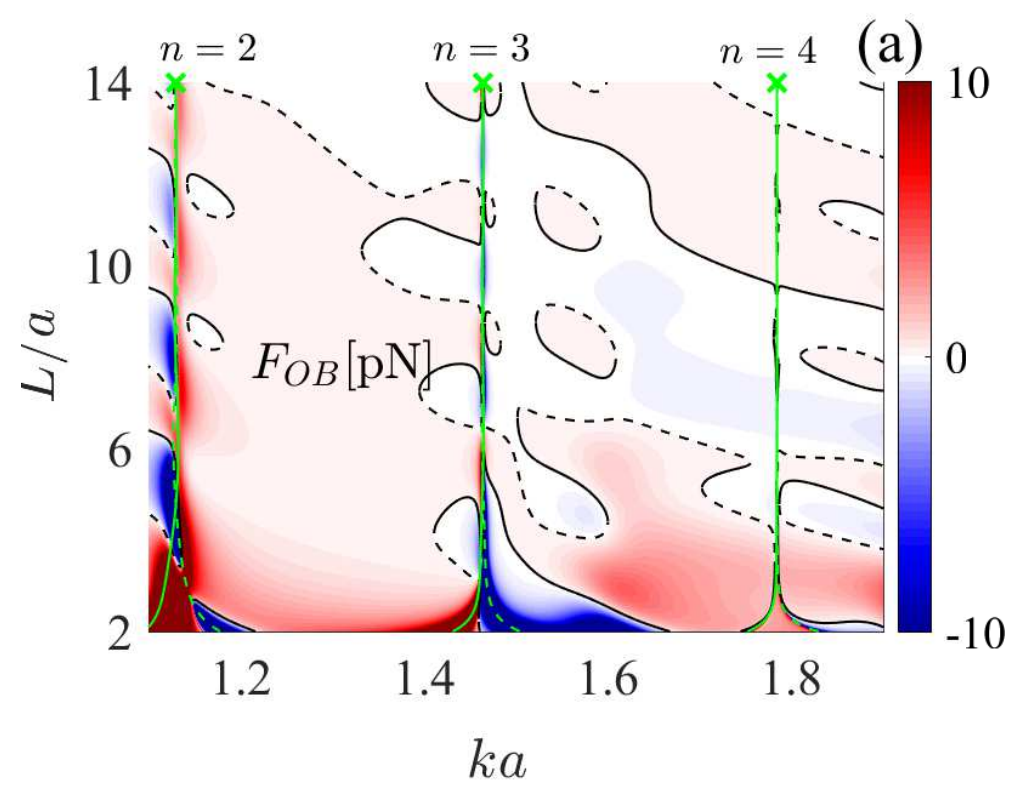

(b) $n=2$

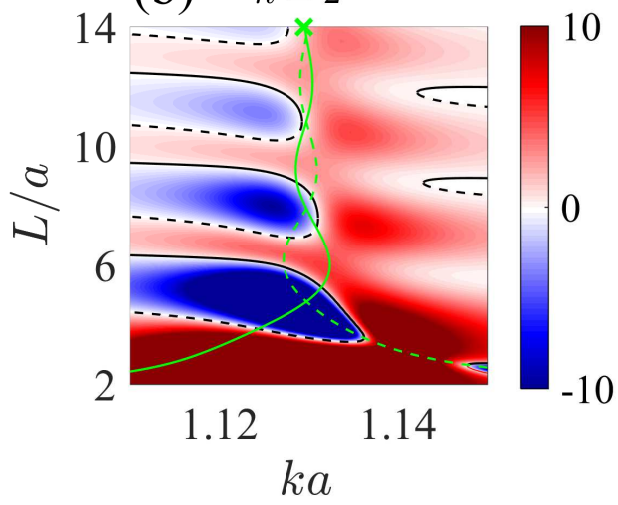

(c)

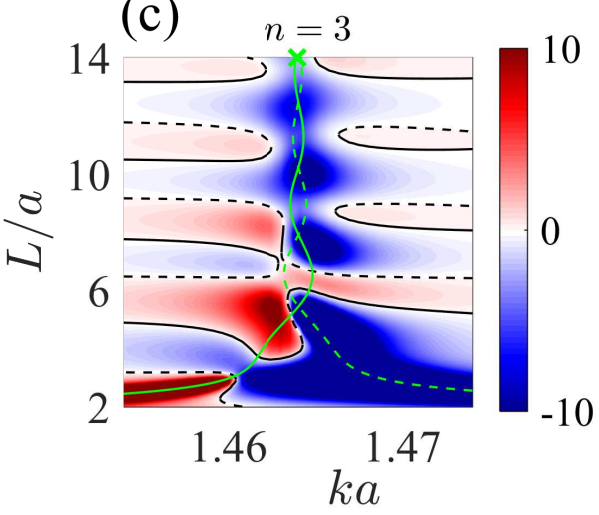

FIG. 3: The same as in Fig. 2 but for $k_{z} a=1$.

between spheres which will be analyzed below by the use of multiple scattering theory. The second peculiarity is the decrease of the resonant $\mathrm{OB}$ force with the order of the Mie resonance $n$. We consider roughly that the more $n$ is the less is the coupling of the Bessel beam for given $k_{z}$ with the Mie resonant mode.

As it was said above the case of two spheres enables analytical treatment of the OB force in the resonant approximation. Owing to the axial symmetry of the total system of two spheres and applied Bessel beam we can take $m=0$ with only three components of EM field $E_{\phi}, H_{r}, H_{\theta}$ for TE polarization in spherical system. Then outside the spheres the EM fields scattered by the spheres can be presented as a series in the vectorial spherical harmonics as follows [36]

$$
\mathbf{E}(\mathbf{r})=\sum_{n} \sum_{j=1,2} b_{n}^{(j)} \mathbf{M}_{n 0}^{(3)}\left(\mathbf{r}-\mathbf{r}_{\mathbf{j}}\right)
$$

where $\mathbf{r}_{j}$ are positions of centers of spheres,

$$
\mathbf{M}_{n 0}^{(3)}(\mathbf{r})=\mathbf{X}_{n 0}(\theta, \phi) h_{n}(k r)
$$

where $\mathbf{X}_{n 0}(\theta, \phi)$ are the vector spherical harmonics [37], $h_{n}(z)$ are the Hankel functions. Here and below the angular index $m=0$ in $b_{n 0}^{(j)}$ is omitted. For the case of the single sphere the optical forces were explicitly derived by Barton 
et al [23] in general case. For the present particular case $m=0$ the $\mathrm{z}$-th component of optical force acting on the first sphere equals

$$
F_{z}^{(1)}=-F_{0} \operatorname{Im} \sum_{n} f_{n}\left[2 b_{n+1}^{(1)} b_{n}^{(1) *}+b_{n+1}^{(1)} B_{n}^{(1) *}+b_{n}^{(1) *} B_{n+1}^{(1)}\right]
$$

where $b_{n}^{(1)}$ are the coefficients of series (2) $, F_{0}=\frac{a^{4} k^{2} E_{0}^{2}}{4 \pi}, \quad f_{n}=\frac{n(n+1)(n+2)}{\sqrt{(2 n+1)(2 n+3)}}$ and

$$
b_{n}^{(j)}=S_{n}(k) B_{n}^{(j)}, j=1,2
$$

where the so-called Lorenz-Mie coefficients are given by

$$
S_{n}(k)=\frac{j_{n}(\sqrt{\epsilon} k a)\left[r j_{n}(k r)\right]_{r=a}^{\prime}-j_{n}(k a)\left[r j_{n}(\sqrt{\epsilon} k r)\right]_{r=a}^{\prime}}{h_{n}(k a)\left[r j_{n}(\sqrt{\epsilon} k r)\right]_{r=a}^{\prime}-j_{n}(\sqrt{\epsilon} k a)\left[r h_{n}(k r)\right]_{r=a}^{\prime}},
$$

$j_{n}(x)$ are the spherical Bessel functions. The case of two spheres was developed by Thanopulos et al [13]. In contrast to Ref. [13] we reveal that the OB force is basically focused around the Mie resonances for high index particles. That prompts to use the resonant approximation which substantially simplifies analysis because of elimination of sum over the orbital indices $n$. Thus, we can truncate the series in Eq. (4) with a preservation of only resonant term given by the Lorenz-Mie coefficient $S_{n}(k)$ around the n-th Mie resonance. We have for the optical force acting on the $j$-th sphere

$$
\frac{F_{z n}^{(j)}}{F_{0}} \approx \sum_{\sigma= \pm 1}(-1)^{(1+\sigma) / 2} f_{n+(\sigma-1) / 2} \operatorname{Im}\left[S_{n}(k)^{*} B_{n+\sigma}^{(j)} B_{n}^{(j) *}\right], \quad \sigma= \pm 1
$$

The incident fields radiating the first sphere is superposed of the incident Bessel beam and the field scattered by the second sphere

$$
\begin{gathered}
B_{n}^{(1)}=B_{n}^{(i n c)}+B_{n, n}^{(21)}(L) b_{n}^{(2)}, \\
B_{n+\sigma}^{(1)}=B_{n+\sigma}^{(i n c)}+B_{n, n+\sigma}^{(21)}(L) b_{n}^{(2)} .
\end{gathered}
$$

Due to the addition theorem [37, 38] we can write the contribution of the second sphere as follows

$$
\begin{gathered}
B_{n, n}^{(21)}(L)=4 \pi \sum_{p=0,2,4, \ldots}^{2 n} g_{n n p} i^{p} \mathcal{G}(n 0 ; n 0 ; p) Y_{p}^{0}(1) h_{p}(k L), \\
B_{n, n+1}^{(21)}(L)=4 \pi \sqrt{\frac{n}{(n+2)}} \sum_{p=1,3,5, \ldots}^{2 n+\sigma} g_{n, n+1, p} i^{p} \mathcal{G}(n 0 ; n+1,0 ; p) Y_{p}^{0}(1) h_{p}(k L),
\end{gathered}
$$

where the argument 1 of Legandre polynomials is related to the direction of scattered field. Substituting the specific expressions for the spherical Bessel functions into Eq. (9) with account of coefficients $g$ and $\mathcal{G}$ tabulated in Ref. 37] we find for $k L \gg 1$

$$
B_{n, n}^{(21)}(L) \approx-c_{n, n} \frac{e^{i k L}}{(k L)^{2}}, \quad B_{n, n+\sigma}^{(21)}(L) \approx-i c_{n, n+\sigma} \frac{e^{i k L}}{(k L)^{2}},
$$

where $c_{1,1}=3, c_{2,2}=15, c_{1,2}=6.708, c_{2,3}=25.1, \ldots$ are real coefficients.

For the large $k L$ we can rewrite Eq. (8) as follows

$$
\begin{gathered}
B_{n}^{(1)} \approx B_{n}^{(i n c)}\left(1+B_{n, n}^{(21)}(L) S_{n}(k) e^{i k_{z} L}\right), \\
B_{n+\sigma}^{(1)} \approx B_{n+\sigma}^{(i n c)}+B_{n, n+\sigma}^{(21)}(L) S_{n}(k) B_{n}^{(i n c)} e^{i k_{z} L} .
\end{gathered}
$$

where we took into account that the Bessel beam (11) accumulates the phase factor $e^{i k_{z} L}$ when reaches the second sphere. Substituting here asymptotes (10) and using an inequality $\left|B_{n}^{(i n c)}\right| \gg\left|B_{n, n}^{(21)}(L) b_{n}^{(2)}\right|$ we can approximate

$$
\begin{gathered}
B_{n}^{(1)} \approx B_{n}^{(i n c)}\left[1-\frac{S_{n}(k)}{(k L)^{2}} c_{n, n} e^{i\left(k_{z}+k\right) L}\right], \\
B_{n+\sigma}^{(1)} \approx B_{n+\sigma}^{(i n c)}-\frac{i S_{n}(k)}{(k L)^{2}} c_{n, n+\sigma} B_{n}^{(i n c)} e^{i\left(k_{z}+k\right) L},
\end{gathered}
$$


As a result we obtain the following expression for the optical force (7) onto the first sphere

$$
\begin{gathered}
F_{z n}^{(1)} \approx F_{0} \sum_{\sigma= \pm 1}(-1)^{(1+\sigma) / 2} f_{n+(\sigma-1) / 2}\left[\operatorname{Im}\left(S_{n}^{*} B_{n}^{(i n c) *} B_{n+\sigma}^{(i n c)}\right)\right. \\
\left.-\frac{c_{n, n}}{(k L)^{2}}\left|S_{n}^{2} B_{n}^{(i n c)} B_{n+\sigma}^{(i n c)}\right| \sin \left(\left(k+k_{z}\right) L+\phi_{n+\sigma}\right)-\frac{c_{n, n+\sigma}}{(k L)^{2}}\left|B_{n}^{(i n c)} S_{n}\right|^{2} \cos \left(k+k_{z}\right) L\right] .
\end{gathered}
$$

where $\phi_{n+\sigma}=\operatorname{Arg}\left(B_{n}^{(i)} B_{n+\sigma}^{(i n c) *} S_{n}(k)^{2}\right)$. Similarly, we have for the second sphere

$$
\begin{gathered}
B_{n}^{(2)}=B_{n}^{(i n c)} e^{i k_{z} L}+B_{n, n}^{(12)}(L) b_{n}^{(1)}, \\
B_{n+\sigma}^{(2)}=B_{n+\sigma}^{(i n c)} e^{i k_{z} L}-B_{n, n+\sigma}^{(12)}(L) b_{n}^{(1)} .
\end{gathered}
$$

By use of identities

$$
B_{n, n}^{(21)}=B_{n, n}^{(12)}, B_{n, n+\sigma}^{(21)}=-B_{n, n+\sigma}^{(12)}
$$

and Eq. (10) we can rewrite Eq. (14) as follows

$$
\begin{gathered}
B_{n}^{(2)}=B_{n}^{(i n c)}\left[e^{i k_{z} L}-\frac{c_{n, n}}{(k L)^{2}} S_{n} e^{i k L}\right], \\
B_{n+\sigma}^{(2)}=B_{n+\sigma}^{(i n c)} e^{i k_{z} L}+\frac{i c_{n, n+\sigma}}{(k L)^{2}} S_{n} B_{n}^{(i n c)} e^{i k L} .
\end{gathered}
$$

As a result we have for the force acting on the second sphere

$$
\begin{gathered}
F_{z n}^{(2)} \approx F_{0} \sum_{\sigma= \pm 1}(-1)^{(1+\sigma) / 2} f_{n+(\sigma-1) / 2}\left[\operatorname{Im}\left(S_{n}^{*} B_{n}^{(i n c) *} B_{n+\sigma}^{(i n c)}\right)\right. \\
\left.+\frac{c_{n, n}}{(k L)^{2}}\left|S_{n}^{2} B_{n}^{(i n c)} B_{n+\sigma}^{(i n c)}\right| \sin \left(\left(k-k_{z}\right) L-\phi_{n+\sigma}\right)+\frac{c_{n, n+\sigma}}{(k L)^{2}}\left|B_{n}^{(i n c)} S_{n}\right|^{2} \cos \left(k-k_{z}\right) L\right],
\end{gathered},
$$

i.e.,

$$
F_{z n}^{(2)}\left(k_{z}\right)=-F_{z n}^{(1)}\left(-k_{z}\right)
$$

Therefore, the asymptotes at $k L \gg 1$ for OB force owing to the dual Bessel beams propagating along the z-axis equal

$$
\begin{gathered}
F_{O B}(L)=F_{z n}^{(1)}\left(k_{z}\right)-F_{z n}^{(2)}\left(k_{z}\right) \approx F_{0} \sum_{\sigma= \pm 1}(-1)^{(1+\sigma) / 2} f_{n+(\sigma-1) / 2} \\
\frac{c_{n, n}}{(k L)^{2}}\left|S_{n}^{2} B_{n}^{(i n c)} B_{n+\sigma}^{(i n c)}\right|\left[\sin \left(\left(k+k_{z}\right) L+\phi_{n+\sigma}\right)+\sin \left(\left(k-k_{z}\right) L+\phi_{n+\sigma}\right)\right]+ \\
\frac{c_{n, n+\sigma}}{(k L)^{2}}\left|B_{n}^{(i n c)} S_{n}\right|^{2}\left[\cos \left(k+k_{z}\right) L+\cos \left(k+k_{z}\right) L\right] .
\end{gathered}
$$

This expression shows two properties of the OB for long distances between spheres: the long-distance and shortrange modulation of the binding force $\frac{2 \pi}{k-k_{z}}$ and $\frac{2 \pi}{k+k_{z}}$ that was reported by Karasek el al [25] numerically by use of a coupled dipole method. It is worthy to note that first the oscillatory behavior of the OB was observed already by Burns et al [2] that was used for separation of $1.43 \mu \mathrm{m}$ polystyren particles in water. An asymptotical decline $1 / L^{2}$ of the $\mathrm{OB}$ force can be also understood if we consider the scattered field from the second sphere positioned at the the z-axis at the distance $L$ is given by the vector spherical function [37]

$$
\mathbf{M}_{n 0}\left(\mathbf{r}-\mathbf{e}_{z} L\right)=-\mathbf{e}_{\phi} h_{n}(k L) \frac{d P_{n}^{0}(\cos \theta)}{d \theta} .
$$

For integration over the first sphere positioned at the $z=0$ the contribution of the second sphere is proportional to $\sin \theta=a / L$. As a result together with the asymptotic of the Bessel function $h_{l}(k L) \sim \frac{e^{i k L}}{k L}$ we obtain the total asymptotic $1 / L^{2}$. We notice that this asymptotic is justified only for coaxial illumination of spheres by the Bessel beams.

The behavior of $\mathrm{OB}$ at the close vicinity of spheres $L \rightarrow 2 a$ is more dramatic as Fig. 4 demonstrates. In order to analytically evaluate this behavior we employ the multiple scattering theory which reduces the Maxwell equations into the linear algebraic equations for the amplitudes $b_{n}$ in expansions of EM fields over vectorial spherical harmonics (2) given by the index $n$ and $m=0$ which can be written as matrix equation

$$
\widehat{L}(k) \vec{\psi}=\vec{\psi}_{\text {inc }}
$$



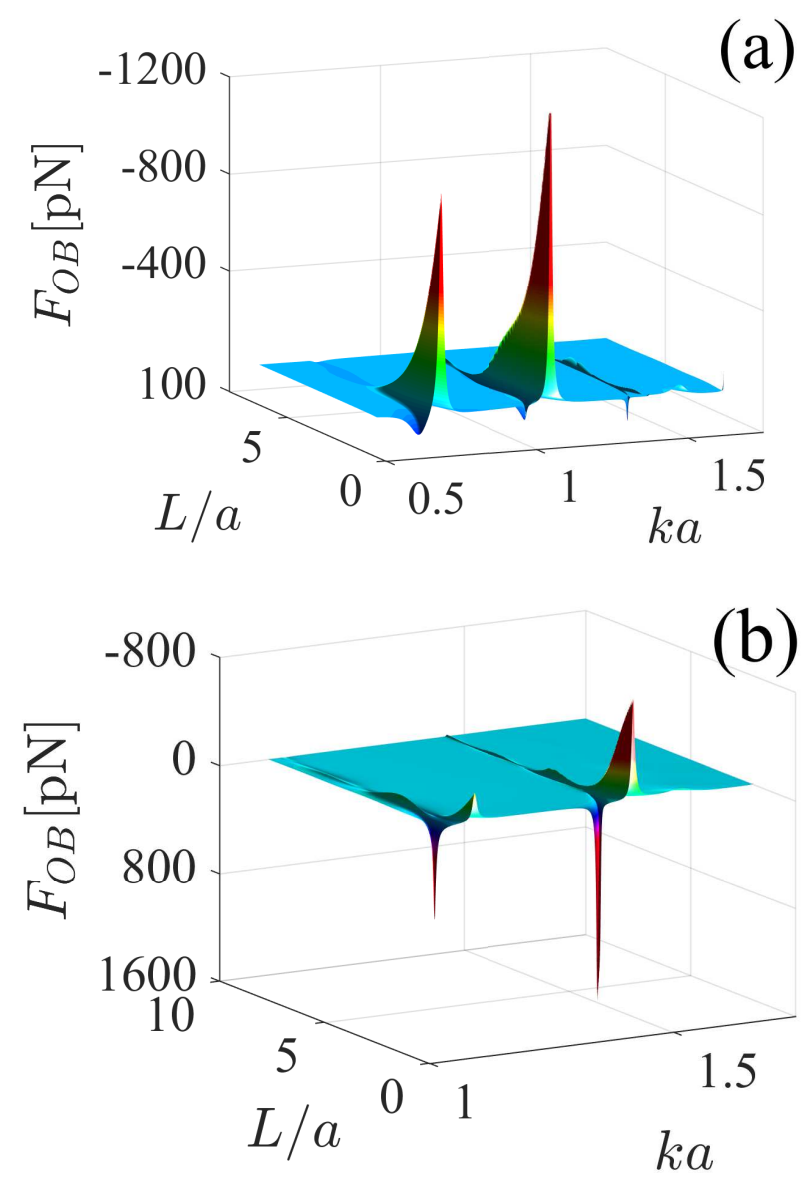

FIG. 4: The binding force between two spheres vs the wavelength and distance for (a) $k_{z} a=1 / 2$ and (b) $k_{z} a=1$.

where the non Hermitian, non symmetric matrix $\widehat{L}(k)$ is determined by a specific structure of dielectric particles. The incident state $\vec{\psi}_{i n c}$ is the column of amplitudes $B_{n}^{(i n c)}$ in this representation. The resonances are given by the solutions of the homogeneous equation

$$
\widehat{L}(k) \vec{\psi}=0
$$

for complex eigenvalues $k$ whose real parts are shown by solid and dash lines in Figs. 2 (b) and (c). For the present problem it is important to note that the matrix $\widehat{L}(k)$ can be defined in the basis of left and right eigenvectors

$$
\vec{y}_{\lambda} \widehat{L}(k)=\lambda \vec{y}_{\lambda}, \quad \widehat{L}(k) \vec{x}_{\lambda}=\lambda \vec{x}_{\lambda},
$$

where $\vec{y}_{\lambda} \vec{x}_{\lambda^{\prime}}=\delta_{\lambda, \lambda^{\prime}}$. By use the condition of completeness

$$
\sum_{\lambda} \vec{x}_{\lambda} \vec{y}_{\lambda}=1
$$

we write the following equalities

$$
\widehat{L}(k)=\sum_{\lambda} \lambda \vec{x}_{\lambda} \vec{y}_{\lambda}, \quad \widehat{L}^{-1}(k)=\sum_{\lambda} \frac{\vec{x}_{\lambda} \vec{y}_{\lambda}}{\lambda} .
$$

as well as for the solution of Eq. (21)

$$
\vec{\psi}=\sum_{\lambda} \frac{W_{\lambda}}{\lambda} \vec{x}_{\lambda}
$$




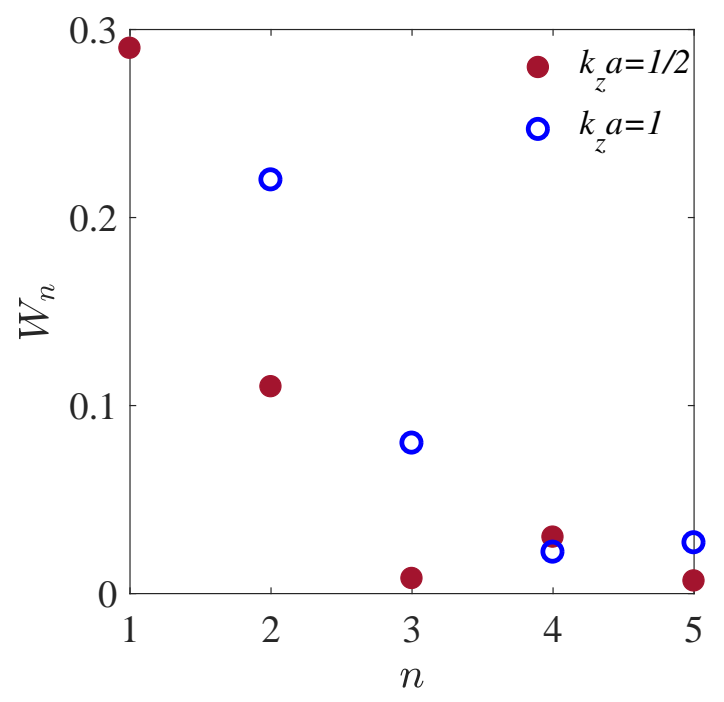

FIG. 5: The coupling coefficient $W_{n}\left(k, k_{z}\right)$ vs the order of Mie resonance $n$.

where

$$
W_{\lambda}=\vec{y}_{\lambda} \vec{\psi}_{i n c}
$$

are the coupling coefficients of the incident wave with the eigenmodes of the open system. For the case of high refractive index of dielectric sphere the index $\lambda$ can be related to those resonant term which has the smallest $\lambda_{n}$ in the vicinity of the resonant frequency $k \approx \operatorname{Re}\left(k_{n}\right)$. That allows to write in the vicinity of the $n$-th Mie resonant frequency $\operatorname{Re}\left(k_{n}\right)$ the eigenvalue as

$$
\lambda_{n}=q_{n}\left(k-k_{n}\right) .
$$

For the case of identical high index particles resonant modes can be presented as symmetric (bonding) and antisymmetric (anti-bonding) modes [39]

$$
\mathbf{E}_{n} \approx \frac{E_{0} W_{n, s}}{\left(k-k_{n, s}\right)} \mathbf{E}_{n, s}+\frac{E_{0} W_{n, a}}{\left(k-k_{n, a}\right)} \mathbf{E}_{n, a},
$$

where the factors $1 / q_{n}$ are absorbed by the coupling constants $W_{n, s, a}$,

$$
\mathbf{E}_{n, s, a}(\mathbf{r}) \approx \frac{1}{\sqrt{2}}\left[\mathbf{M}_{n 0}\left(\mathbf{r}-\frac{L}{2} \mathbf{e}_{z}\right) \pm \mathbf{M}_{n 0}\left(\mathbf{r}+\frac{L}{2} \mathbf{e}_{z}\right)\right]
$$

$E_{0}$ is the amplitude of the Bessel beam. The coupling constant of incident Bessel beam (10) with the symmetric or anti-symmetric resonant modes (30) can according to the definition (27) be presented as (see also [40])

$$
W_{n, s, a} \approx W_{n}\left(k, k_{z}\right)\left\{\begin{array}{c}
\cos k_{z} L / 2 \\
i \sin k_{z} L / 2
\end{array}\right.
$$

where $W_{n}\left(k, k_{z}\right)$ is the coupling constant of the Bessel beam with the $n$-th Mie resonant mode. One can perform analytical calculations of the constant by the use of a great deal of algebra presented in Refs. 34, 35, 41, 42]. However it is simpler to find the coupling constants numerically because their values are independent on the distance. The results are presented in Fig. 5 for $k_{z} a=0.5,1$ and show that the OB force decreases with $n$.

The resonant frequencies in the two-level approximation can be written as follows [39]

$$
k_{n, s, a}=\operatorname{Re}\left(k_{n, s, a}\right)-i \gamma_{n, s, a} \approx k_{n} \pm \frac{v_{n}}{L^{2}} e^{i\left(k_{n} L-\theta_{n}\right)} .
$$

Fig. [6 shows that the resonant frequencies (32) well describes numerically calculated dipole resonances $n=1$ with fitting parameters $v_{1}=0.15$ and $\theta_{1}=1.25$. As seen from Figs. 2 and 3 at close distances between spheres the bonding and anti-bonding resonances are well separated that allows us to consider them independently. In what follows we consider in details the anti-bonding dipole resonance $n=1$ for which the OB noticeably exceeds the case 

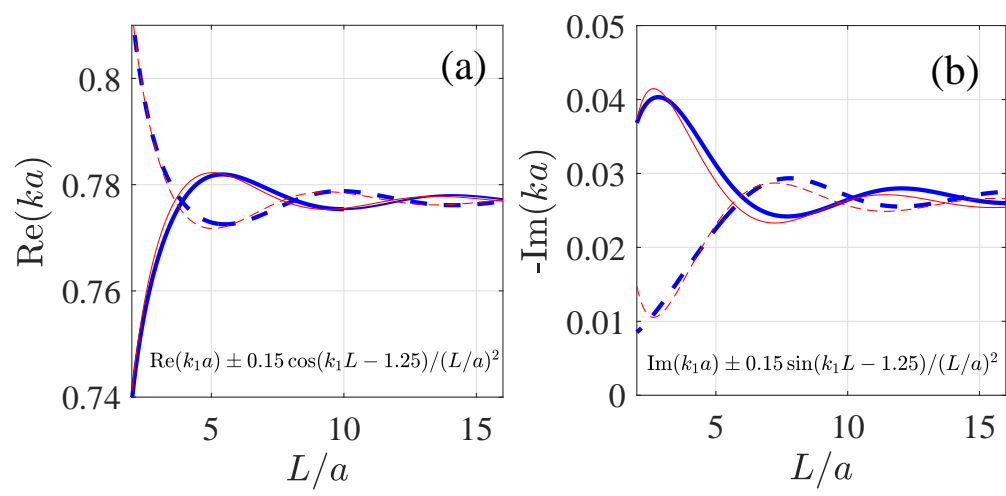

FIG. 6: The dipole $n=1$ resonant frequencies of two coupled spheres, real (a) and imaginary (b) vs the distance calculated from Eq. (22) (thick blue) and compared to two-level approximation (32) (thin red) and presented with fitting parameters in Figures. Solid (dash) lines show the frequencies of bonding (anti-bonding) resonances.

(a)

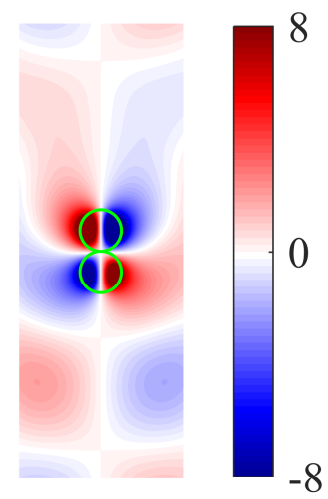

(b)

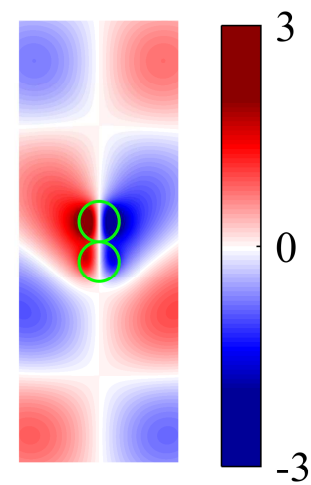

FIG. 7: Numerically computed scattered field (the component $E_{\phi}$ ) at the closest distance $L=2 a$ for frequencies around the dipole Mie resonance $k_{1}$ : (a) $k=\operatorname{Re}\left(k_{1, a}\right)$ (anti-bonding resonant mode), (b) $k=\operatorname{Re}\left(k_{1, s}\right)$ (bonding resonant mode) The Bessel beam with $k_{z} a=0.5$ illuminates spheres from bottom.

of the bonding resonance as Fig. (4 (a) shows. The reason is related to the denominators in Eq. (29) which equal the imaginary parts of the resonances $\operatorname{Im}\left(k_{n, s, a}\right)$ at $k=\operatorname{Re}\left(k_{n, s, a}\right)$. Other words, the near fields are proportional to the quality factors $Q_{n, s, a}=-\operatorname{Re}\left(k_{n, s, a}\right) / 2 \operatorname{Im}\left(k_{n, s, a}\right)$. For the dipole case resonances with $n=1$ the $Q_{1, s} \rightarrow 10$ while $Q_{1, a} \rightarrow 56$ at $L \rightarrow 2 a$. The response of the scattered field around the anti-bonding resonance becomes strong compared to the incident Bessel beam. Therefore the incident field can be neglected. Fig. 7 (a) demonstrates that the scattered field indeed slightly differs from the antisymmetric mode $\vec{E}_{n, a}$ given by Eq. (30). That directly correlates with the behavior of the resonant width vs $L$ shown in Fig. 6 (b). One can see that $\operatorname{Im}\left(k_{1, a}\right)$ has a minimum at $L \approx 2 a$. While Fig. 7 (b) shows that the Bessel beam contributes significantly into the scattered field when $k \approx k_{1, s}$ and therefore can not be disregarded. That is a consequence of the resonant width of the anti-bonding dipole resonant mode $1, s$. One can see from Fig. 6 (b) that the resonant width of the bonding dipole resonant mode $1, s$ reaches maximum for $L \rightarrow 2 a$.

Comparison of Eq. (29) with Eq. (5) gives us

$$
b_{n}^{(j)}=(-1)^{j-1} E_{0} d_{n}, j=1,2,
$$



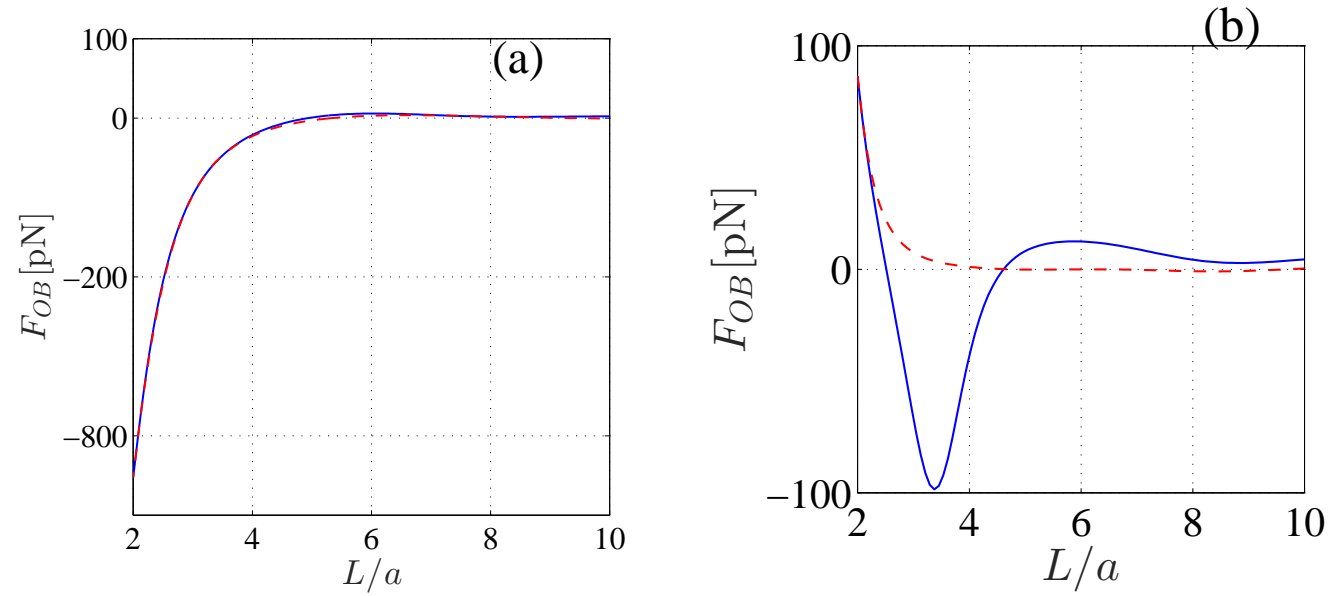

FIG. 8: The OB vs distance between spheres at the vicinity of the dipole anti-bonding (a) resonance $k=\operatorname{Re}\left(k_{1, a}(L)\right)$ and $(\mathrm{b})$ bonding resonance $k=\operatorname{Re}\left(k_{1, s}(L)\right)$. The solid line shows numerics and dash line shows approximated formulas. Wave number of Bessel beam $k_{z} a=1 / 2$.

where $d_{n}=\frac{W_{n, a}}{\sqrt{2} q \gamma_{n, a}} \sin \left(k_{z} L / 2\right)$. Owing to Eqs. (5) and (8) we have

$$
\begin{gathered}
B_{n}^{(1)} \approx d_{n}\left(\frac{1}{S_{n}\left(k_{n}\right)}-B_{n, n}^{(21)}(L)\right), \\
B_{n+1}^{(1)} \approx B_{n, n+1}^{(21)}(L) b_{n}^{(2)}=-d_{n} B_{n, n+1}^{(21)}(L) .
\end{gathered}
$$

According to Eq. (4) we obtain for the force acting onto the first sphere around the anti-bonding dipole resonance $k \approx \operatorname{Re}\left(k_{1, a}\right)$ :

$$
\frac{F_{z 1}^{(1)}}{F_{0}} \approx-\operatorname{Im}\left(S_{1}^{*} B_{1}^{(1) *} B_{2}^{(1)}\right) \approx-\left|d_{1}\right|^{2} \operatorname{Im}\left[\left(1-S_{1} B_{1,2}^{(21) *}(L)\right)\left(1+B_{1,1}^{(21) *}(L)\right)\right]
$$

where

$$
B_{1,1}^{(21)}(L)=h_{0}(k L)+h_{2}(k L), B_{1,2}^{(21)}(L)=-1.3416\left(h_{1}(k L)+h_{3}(k L)\right)
$$

owing to Eq. (9). Taking into account relations (15) we obtain the OB at $L \geq 2 a$

$$
\frac{F_{O B}}{F_{0}}=2 \frac{F_{z 1}^{(1)}}{F_{0}}=\frac{1.3416 f_{1}\left|W_{1}\right|^{2} \sin ^{2}\left(k_{z} L / 2\right)}{\gamma_{1, a}^{2}(L)} \operatorname{Im}\left[\left(1-S_{1}^{*}\left(k_{1, a}\right)\right)\left(h_{0}^{*}(k L)+h_{2}^{*}(k L)\right)\left(h_{1}(k L)+h_{3}(k L)\right)\right] .
$$

Fig. 8(a) shows the asymptotic formula (36) perfectly describes the numerically computed OB force for the dipole anti-bonding resonance. Similar asymptotic formula can be written for the bonding resonance by simple substitution $a \rightarrow s$. However Fig. 8 (b) shows strong discrepancy between numerics and asymptotic formula. The discrepancy is related to that as seen from Fig. 6 (b) the bonding resonant width reaches maximum at $L \rightarrow 2 a$. As a result enhancement of scattered EM field at the bonding resonance roughly four times yields to the case of anti-bonding resonance. Therefore for calculation of the optical forces we can not neglect the incident fields as distinct from the dipole anti-bonding resonance.

Next, with growth of the order of the Mie TE resonances $n$ in the dielectric sphere the resonant width exponentially decreases [43, 44]. Therefore one could expect the fast growth of the OB force. However by the same reason of reduction of radiation losses with $n$ decrease of the coupling of the Mie resonant modes with the Bessel beam occurs that Fig. 5 demonstrates.

Moreover two parameters, the frequency and by wave vector $k_{z}$ along the propagation axis $z$ define the Bessel beam (11). Figs. 2 and 3 show that indeed these parameters noticeably effect the equilibrium distances between the spheres. Eq. (36) predicts simple dependence of the $\mathrm{OB}$ on the longitudinal wave number $k_{z}$ of the Bessel beam in the form of 

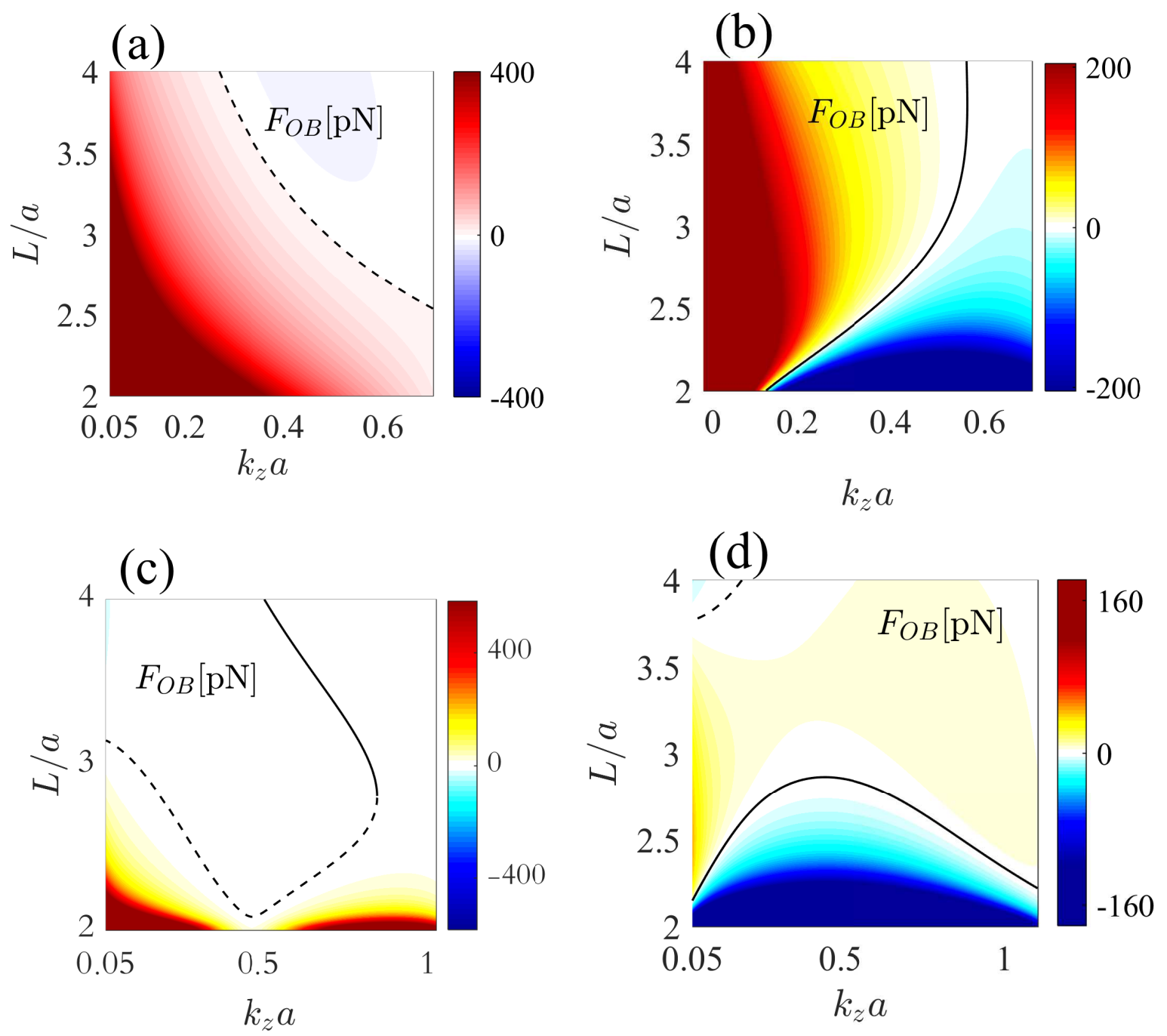

FIG. 9: The $\mathrm{OB}$ vs distance between spheres and longitudinal wave number of the Bessel beam at the vicinity of the TE Mie resonances. (a) $k a=0.735$ around the bonding dipole resonance $n=1$, (b) $k a=0.82$ around the anti-bonding dipole resonance $n=1$, (c) $k a=1.0947$ around the bonding quadruple resonance $n=2$, and (d) $k a=1.1783$ around the anti-bonding quadruple resonance at $L=2 a$. The solid line shows equilibrium positions of spheres.

$\sin ^{2} k_{z} L / 2$ but rather complicated dependence on the distance $L$ through the Hankel functions for the anti-bonding dipole resonance. This conclusion is illustrated in Figs. 9 which shows strong dependence of the OB force on $k_{z}$ and $L$ for frequencies tuned to the dipole and quadruple anti-bonding frequencies $\operatorname{Re}\left(k_{1, a}\right)$ and $\operatorname{Re}\left(k_{2, a}\right)$, respectively. One can see that these results provide potentially useful way to manipulate distance between particles by variation of the longitudinal wave number of the Bessel beams.

\section{OPTICAL BINDING FORCE BETWEEN TWO COAXIAL DISKS}

Distinct to the case of two spheres, two disks have two parameters to vary, the aspect ratio and distance between disks. Even in an isolated dielectric disk the high-Q resonances can be achieved by avoided crossing of the TE resonances of the same symmetry relative to inversion of the disk's axis under variation of the aspect ratio around $a / h=0.71$ as it was reported by Rybin et al [45] and illustrated in Fig. 10 (a) and (b). While the resonances of the opposite symmetry in an isolated disk plotted by solid and dash lines can not be coupled in single disk. An example of this crossing is highlighted by circle in Fig. 10 (a). However, the presence of the second disk lifts this symmetry restriction giving rise to a new series of avoided crossings of resonances shown in Fig. 10 (c) 22]. In view of the OB force the most important is the anti-bonding resonance which achieves unprecedent high $Q$ factor around 18000 as 
shown in Fig. 10 (d). The reason of such an extreme value is related to that the anti-bonding resonant mode is close to the Mie resonant mode with extremely large orbital index $(n=6)$ of an effective sphere with the volume equal $\pi(h+L) a^{2}$ shown in right bottom inset of Fig. 10 (c) 22]. That refers also to the bonding resonant mode which is close to the Mie resonant mode with $n=5$ shown in left bottom inset in Fig. 10 (c). Respectively we expect around the aspect ratio $a / h=1$ extremal enhancement of $\mathrm{OB}$, especially for the anti-bonding resonant mode similar to Refs. [14 16]. These effective spheres are shown by white lines in bottom insets.
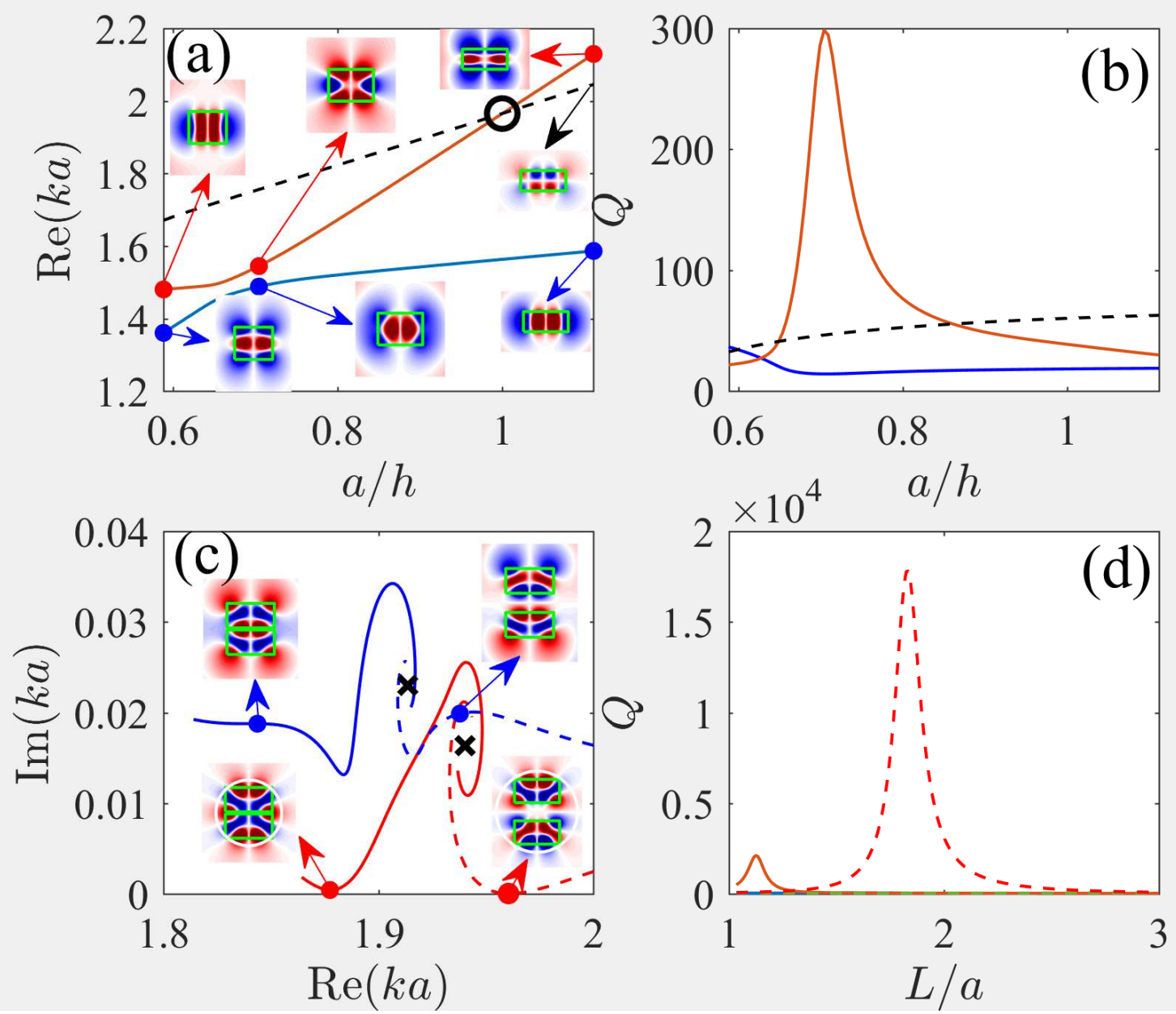

FIG. 10: (a) Avoided crossing of two TE resonances whose modes are symmetric relative to $z \rightarrow-z$ for traversing over distance and (b) their $Q$ factors versus the aspect ratio $a / h$ in isolated silicon disk. (c) Behavior of hybridized resonances and (d) the $Q$ factor vs distance between centers of disks for $a / h=1.003$. Insets show the profiles of tangential component of electric field $E_{\phi}$.

First, we consider a stability of single disk at $r=0$. Numerical calculations of forces by the centered Bessel beam and slightly shifted beam relative to axis $r=0$ have shown that the position of disk is stable at the symmetry axis at the vicinity of resonant frequencies. That considerably simplifies the further calculation of OB between two disks. The results of calculations of the OB are presented in Figs. 11 and 12 Fig. 12 demonstrates that indeed near the parameters of extremely large peaks of the $Q$ factor we observe respectively giant OB of order one femto Newton. For the reader's convenience we reproduce Fig. 11 as surface in Fig. 12 (a) where one can see that giant OB is achieved around 30 femto Newtons at $k a=1.97, L=1.85 a, h=1.03 a, k_{z} a=0.5$. Fig. 12 (b) shows that this giant peak is split for $k_{z} a=1$. It is remarkable that the equilibrium distances between disks is traversed close to the anti-bonding resonance shown by dotted line. That situation was first reported for two dielectric slabs which can move in waveguide that is equivalent to Fabry-Perot resonator with high $Q$ resonances [46]. Fig. [13] demonstrates that these giant peaks are easily manipulated by small changes of parameters of the Bessel beam: $k_{z} a$ and frequency. 


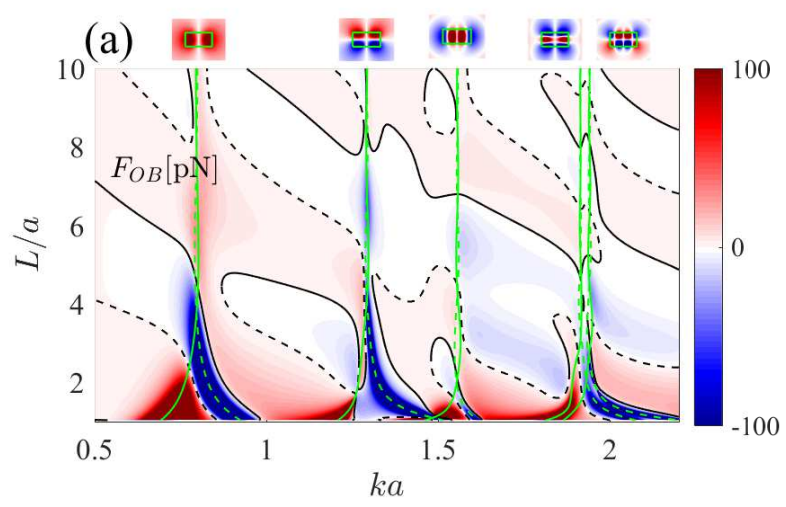

(b)

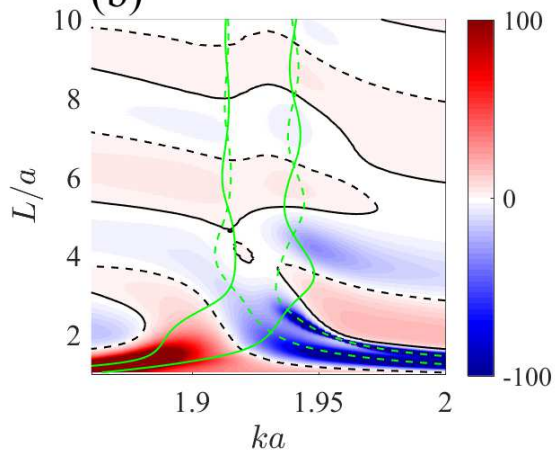

FIG. 11: The binding force between two disks vs the frequency and distance between centers of disks for the Bessel beam with TE polarization and $k_{z} a=1 / 2$ where the disk with $\epsilon=15$ has the radius $a=0.5 \mu m$. (b) zoomed versions. Black solid (dash) lines show stable (unstable) configuration of disks. Light green solid (dash) lines show bonding (symmetric) and anti-bonding (anti symmetric) resonant frequencies of two disks vs the distance between.
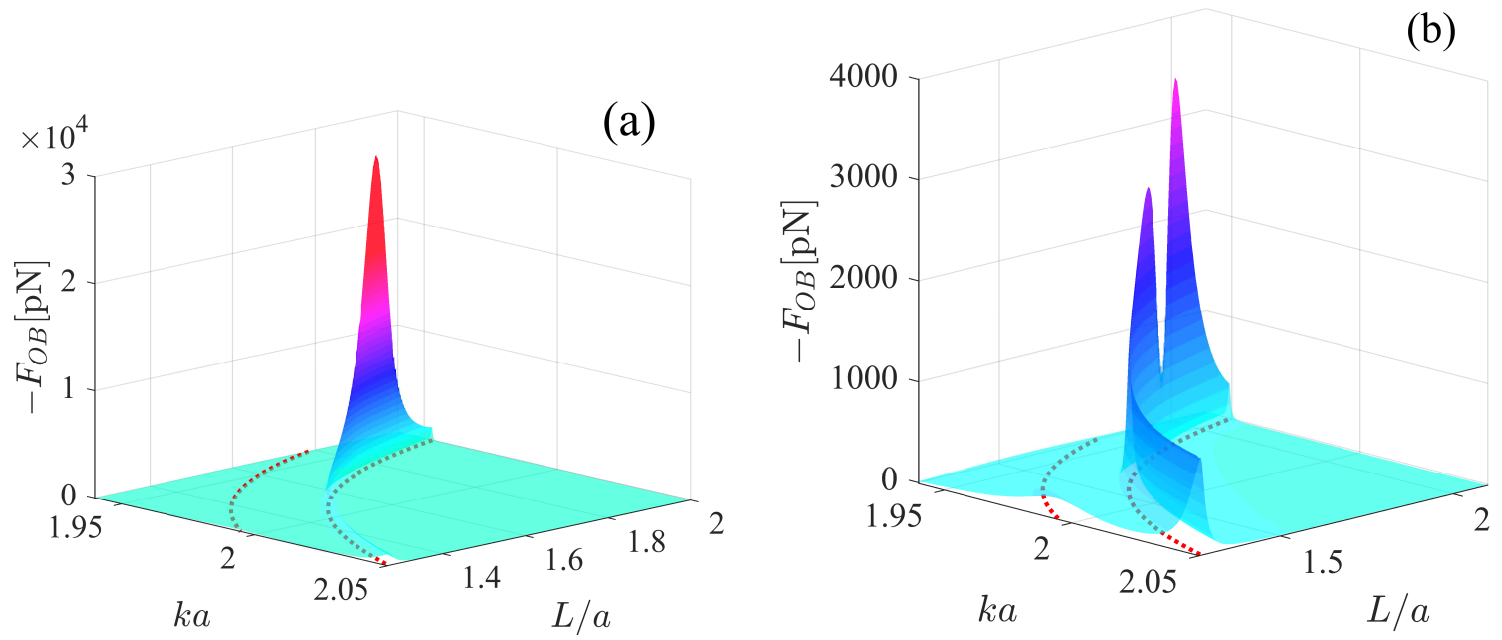

FIG. 12: The OB vs distance between centers of disks at the vicinity of the anti-bonding resonance marked in Fig. 10 (c) by closed circle $k a=1.95$ (a) $k_{z} a=0.5$ and (b) $k_{z} a=1$. Solid line underneath shows resonant frequency vs distance $L$ highlighted in Fig. 11

(a)

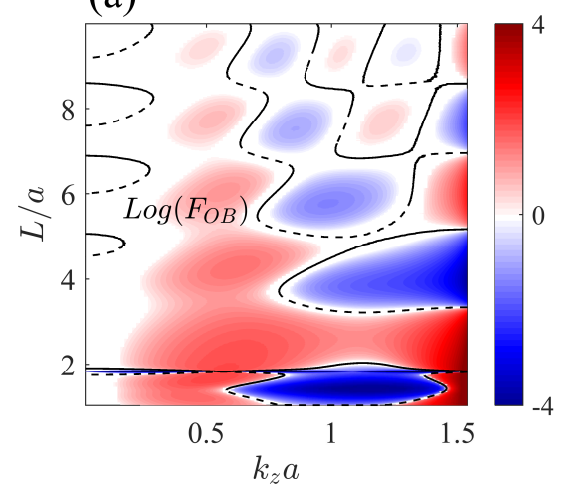

(b)

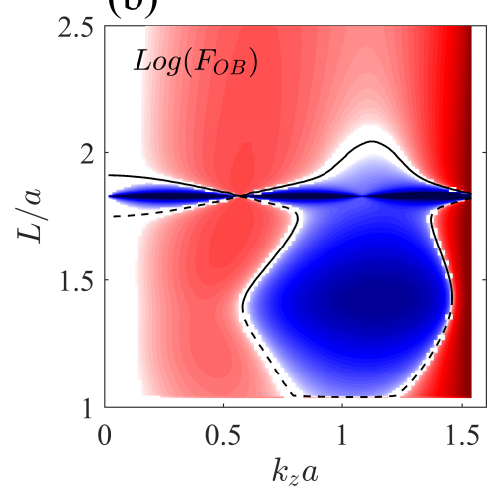

FIG. 13: (a) The OB vs distance between disks and longitudinal wave vector of the Bessel beam $k_{z} a$ at the vicinity of the anti-bonding resonance marked in Fig. 10 (c) by closed circle $k a=1.95$. (b) Zoomed version of (a). 


\section{SUMMARY AND CONCLUSIONS}

In the present paper we consider optical binding $(\mathrm{OB})$ of particles of micron size by illumination of dual counter propagating Bessel beams. The case of two spheres owing to formulas derived by Barton et al [23] for electromagnetic force acting on the isolated sphere gives an opportunity to derive analytical expressions for the OB force in the resonant approximation. At large distances the OB force decays as inverse squared distance and has two periods of oscillations (Eq. (19)). For near distances the OB force can be considerably enhanced up to order of one femto Newton. One of the important and unexpected results of the OB forces of spheres is their decrease with growth of the order of the Mie resonances. That is a result of competition of two types of couplings. The first coupling of the Mie resonant modes of the sphere with the radiation continua given by the vectorial spherical functions fast falls with growth of the order of resonance giving rise to WGMs with extremal $Q$ factors [43, 44]. However the couplings of the Mie resonant modes with the incident Bessel beams can decay even faster with the growth of the order of the Mie resonant mode. Indeed, our calculations presented in Fig. 4 that the OB force is large only for the dipole and quadruple Mie resonances.

The case of coaxial disks brings a new aspect for the OB force related to the extremely high $Q$ factor due to twoparametric avoided crossing of orthogonal resonances over aspect ratio and distance between the disks [22]. For the case of two coaxial silicon disks with micron diameter illuminated by dual coaxial Bessel beams we demonstrate giant OB force in few decades of femto Newtons in the vicinity of anti-bonding resonances. The corresponding anti-bonding resonant mode of two disks is turned out to be close to the Mie resonant mode with high orbital index $n=6$ of an effective sphere of the volume $\pi a^{2}(h+L)$ [22] with extremely high $Q$ factor. That allows to achieve giant OB force around a several decades of femto Newtons.

There are three important aspects of the OB force of two high index dielectric particles. The first is a giant values of the force around one nano Newtons for two spheres and a few decades of nano Newtons for two coaxial disks illuminated by dual Bessel beams with power $1 \mathrm{~mW} / \mu \mathrm{m}$. The second aspect is that the giant OB forces are caused by resonant excitation of subwavelenth resonant modes of particles. Potentially easy way for cardinal manipulation of the OB force by a cross-section of the Bessel beam constitutes the third aspect of presented results.

\section{Acknowledgments}

The work was supported by Russian Foundation for Basic Research projects No. 19-02-00055. AS thanks Dmitrii Maksimov and Evgeny Sherman for helpful discussions.

[1] A. Ashkin, J. M. Dziedzic, J. E. Bjorkholm, and S. Chu, Opt. Lett. 11, 290 (1986).

[2] M. M. Burns, J.-M. Fournier, and J. A. Golovchenko, Phys. Rev. Lett. 63, 1233 (1989).

[3] S. A. Tatarkova, A. E. Carruthers, and K. Dholakia, Phys. Rev. Lett. 89 (2002).

[4] R. Gómez-Medina and J. J. Sáenz, Phys. Rev. Lett. 93 (2004).

[5] N. K. Metzger, K. Dholakia, and E. M. Wright, Phys. Rev. Lett. 96 (2006).

[6] N. K. Metzger, E. M. Wright, and K. Dholakia, New J. Phys. 8, 139 (2006).

[7] K. Dholakia and P. Zemánek, Rev. Mod. Phys. 82, 1767 (2010).

[8] R. Bowman and M. Padgett, Rep. Progr. Phys. 76, 026401 (2013).

[9] I. Thanopulos, D. Luckhaus, and R. Signorell, Phys. Rev. A 95 (2017).

[10] P. C. Chaumet and M. Nieto-Vesperinas, Phys. Rev. B 64 (2001).

[11] J. Ng, Z. Lin, C. Chan, and P. Sheng, Phys. Rev. B 72 (2005).

[12] T. Cižmár, V. Kollárová, Z. Bouchal, and P. Zemánek, New J. Phys. 8, 43 (2006).

[13] I. Thanopulos, D. Luckhaus, T. Preston, and R. Signorell, J. Appl. Phys. 115, 154304 (2014).

[14] M. I. Antonoyiannakis and J. B. Pendry, Europhys. Lett. 40, 613 (1997).

[15] V. Liu, M. Povinelli, and S. Fan, Opt. Express 17 (2009).

[16] J. Zhang, K. MacDonald, and N. Zheludev, Opt. Lett. 39, 4883 (2014).

[17] A. Taghizadeh and I.-S. Chung, Appl. Phys. Lett. 111, 031114 (2017).

[18] Z. F. Sadrieva, M. A. Belyakov, M. A. Balezin, P. V. Kapitanova, E. A. Nenasheva, A. F. Sadreev, and A. A. Bogdanov, Phys. Rev. A 99, 053804 (2019).

[19] E. Bulgakov and A. Sadreev, Phys. Rev. A 99, 033851 (2019).

[20] M. L. Povinelli, S. Johnson, M. Loncar, M. Ibanescu, E. Smythe, F. Capasso, and J. D. Joannopoulos, Optics Express 13, 8286 (2005).

[21] M. Benyoucef, J.-B. Shim, J. Wiersig, and O. G. Schmidt, Opt. Lett. 36, 1317 (2011).

[22] E. N. Bulgakov, K. N. Pichugin, and A. F. Sadreev (2020), 2005.05554v1. 
[23] J. P. Barton, D. R. Alexander, and S. A. Schaub, J. Appl. Phys. 66, 4594 (1989).

[24] V. Karásek and P. Zemánek, Journal of Optics A: Pure and Applied Optics 9, S215 (2007).

[25] V. Karásek, O. Brzobohatý, and P. Zemánek, J. Optics A: Pure and Appl. Optics 11, 034009 (2009).

[26] Y. Zhu, Z. Wu, Z. Li, and Q. Shang, Procedia Engineering 102, 329 (2015).

[27] F. Deng, H. Liu, M. Panmai, and S. Lan, Opt. Express 26, 20051 (2018).

[28] G. Milne, K. Dholakia, D. McGloin, K. Volke-Sepulveda, and P. Zemánek, Opt. Express 15, 13972 (2007).

[29] L.D.Landau and E.M.Lifshitz, Electrodynamics of Continuous Media (Pergamon, New York, 1960).

[30] M. I. Antonoyiannakis and J. B. Pendry, Phys. Rev. B 60, 2363 (1999).

[31] J. Chen, J. Ng, S. Liu, and Z. Lin, Phys. Rev. E 80 (2009).

[32] N. Wang, J. Chen, S. Liu, and Z. Lin, Phys. Rev. A 87 (2013).

[33] S. Song, N. Wang, W. Lu, and Z. Lin, J. Opt. Soc. Am. A 31, 2192 (2014).

[34] A. D. Kiselev and D. O. Plutenko, Phys. Rev. A 94 (2016).

[35] A. Neves and C. Cesar, J. Opt. Soc. Am. B 36, 1525 (2019).

[36] J. Stratton, Electromagnetic theory (McGraw-Hill Book Company, Inc., 1941).

[37] C. Linton, V. Zalipaev, and I. Thompson, Wave Motion 50, 29 (2013).

[38] D. Mackowski, Proceedings of the Royal Society of London. Series A: Math. and Phys. Sciences 433,599 (1991).

[39] K. N. Pichugin and A. F. Sadreev, J. Appl. Phys. 126, 093105 (2019).

[40] Q. Song, M. Zhao, L. Liu, J. Chai, G. He, H. Xiang, D. Han, and J. Zi, Phys. Rev. A 100 (2019).

[41] A. S. van de Nes and P. Torok, Opt. Express 15, 13360 (2007).

[42] Y. Jiang, Y. Shao, X. Qu, J. Ou, and H. Hua, J. Opt. 14, 125709 (2012).

[43] C. C. Lam, P. T. Leung, and K. Young, J. Opt. Soc. Am. B 9, 1585 (1992).

[44] M. L. Gorodetsky, A. A. Savchenkov, and V. S. Ilchenko, Opt. Lett. 21, 453 (1996).

[45] M. Rybin, K. Koshelev, Z. Sadrieva, K. Samusev, A. Bogdanov, M. Limonov, and Y. Kivshar, Phys. Rev. Lett. 119, 243901 (2017).

[46] A. Sadreev and E. Y. Sherman, Phys. Rev. A 94, 033820 (2016). 